\title{
Manifestación de la estructura de dominios en el comportamiento dieléctrico no lineal de una cerámica piezoeléctrica
}

\author{
J.E. GARCíA, R. PÉREZ, A.AlbAREDA \\ Dept. Física Aplicada. U. Politècnica de Catalunya. 08038 Barcelona. Spain
}

\begin{abstract}
Se pretende mejorar la descripción del comportamiento dieléctrico no lineal de cerámicas piezoeléctricas PZT, sometidas a distintas acciones exteriores, para correlacionarlo con los distintos procesos que se desarrollan en él debidos a su estructura. Mediante un puente, construido ad hoc, se analiza la corriente obtenida al aplicar una tensión sinusoidal de gran amplitud y baja frecuencia. Se intenta que en el proceso de datos no se presuponga la validez de ningún modelo. Se observa que el comportamiento no lineal depende explícitamente de la amplitud del campo. El incremento no lineal de la constante dieléctrica puede desdoblarse en dos fracciones. Una de ellas, $\varepsilon_{\alpha}$, no depende del campo instantáneo, siendo mucho mayor en los materiales blandos que en los duros. Es también mayor en cerámicas no envejecidas , o cuya polarización ha sido alterada. Nada de ello ocurre con la fracción restante. Las pérdidas están relacionadas directamente con el término $\varepsilon_{\alpha}$ para todos los tipos de PZT. Es razonable suponer que dicho término aparece a causa del movimiento irreversible de las paredes de dominio. El término $\varepsilon_{\alpha}$ depende de la amplitud del campo a través de una función potencial, cuyo exponente fraccionario depende del tipo de cerámica.
\end{abstract}

Palabras clave: Piezoceramicas, PZT, no-linealidad, constante dieléctrica

Revelation of domain structure through nonlinear dielectric behavior in a piezoelectric ceramic

It has been intended to improve the description of the nonlinear dielectric behavior of piezoelectric ceramic PZT, by applying diverse external actions, in order to correlate it to the phenomena that occur due to its structure. By using a bridge built for this purpose, the current obtained by applying a harmonic high voltage of low frequency is analyzed. It is intended to do not use any previous model in the treatment of the data. It can be seen that the nonlinear behavior depends explicitly on the field amplitude. The nonlinear increment of the dielectric constant can be split into two parts. One of them, $\varepsilon_{\alpha}$, does not depend on the instantaneous field, and its value is higher in soft than in hard materials. It is also higher if the ceramics has been deaged or if its polarization has been altered. None of these effects occurs to the other part of the increment of $\varepsilon$ Furthermore, losses are closely related to the term $\varepsilon_{\alpha}$ for all types of PZT. It is reasonable to expect that this term comes from the nonreversible movement of non- $180^{\circ}$ domain walls. The term $\varepsilon_{\alpha}$ depends on the amplitude $\mathrm{E}_{0}$ through a power function, with a fractional power that depends on ceramic type.

Key words: Piezoceramics, PZT, non linearity, dielectric constant

\section{INTRODUCCIÓN}

En los dispositivos piezoeléctricos de alta potencia, las cerámicas usadas están sometidas a condiciones extremas, en las que su comportamiento dieléctrico y elástico puede ser muy distinto del que se observa al medir en baja señal (1-4). El estudio de esas alteraciones no sólo será de provecho para quien diseña los dispositivos, preocupado por darles la dimensión adecuada y garantizar su fiabilidad, sino que pueden favorecer el futuro desarrollo de nuevos y mejores materiales.

Por ello, es preciso investigar los mecanismos que actúan al aplicar un campo eléctrico alterno e intenso sobre la cerámica. Es sabido que la mayor parte del efecto piezoeléctrico se produce debido al desplazamiento de las paredes de dominios distintas de $180^{\circ}$ (efecto extrínseco) $(5,6)$, que los efectos no lineales están relacionadas con dicho desplazamiento (7) y que en los materiales blandos el factor determinante es la interacción de las paredes con los defectos puntuales (8). Pero en otros materiales aparecen comportamientos de carácter claramente distinto (9), siendo difícil no sólo la interpretación del fenómeno, sino incluso su descripción.
La estructura interna de dominios no sólo se manifiesta en las propiedades lineales de una cerámica piezoeléctrica, sino que los comportamientos no lineales (dieléctrico y elástico) están notablemente condicionados por ella. Debido a su mayor complejidad, debe describirse mediante un número mayor de variables, lo que a su vez permite una descripción mejor del estado de la cerámica.

Debe tenerse en cuenta que el estado de la cerámica depende de muchos factores, que podemos agrupar en tres tipos: las que dependen del proceso de fabricación (composición, dopantes, tamaño de grano), las modificaciones sufridas desde entonces ( polarización, tratamientos térmicos, envejecimiento y fatiga) y las acciones que se están aplicando en este momento (campo y esfuerzo).

Así pues, se asume que las medidas no lineales no son una propiedad exclusiva de un tipo de material a una temperatura dada, sino que dependen de los procesos que ha sufrido el material desde su fabricación, en particular de la forma y las condiciones en que fue polarizado, de las alteraciones severas 
de temperatura que haya sufrido, y en general de las acciones a las que ha sido sometido y del tiempo transcurrido desde su aplicación. Por ello, esas propiedades lo son de una muestra concreta en unas condiciones concretas.

Al describir de forma no lineal una cerámica, se está tratando un sistema complejo, cuya característica principal no consiste solamente en que las relaciones sean distintas al caso lineal, sino que el numero de grados de libertad ha aumentado, multiplicándose el numero de situaciones posibles y aumentando las variables de las que dependen los procesos. Por ello es conveniente identificar unos comportamientos que, aun cuando su interpretación sea todavía lejana, se cumplen de forma manifiesta en todos o en la mayoría de los casos.

\section{PROCEDIMIENTO EXPERIMENTAL}

Las medidas realizadas consisten en la obtención de la corriente que circula por la muestra al aplicar campos eléctricos sinusoidales de baja frecuencia y de distinta amplitud. De hecho, nos interesa medir $\mathrm{I}_{\mathrm{NL}}$ que es la diferencia entre la corriente real y la corriente que correspondería al comportamiento lineal ideal.

Para obtener buena sensibilidad en la medida de $\mathrm{I}_{\mathrm{NL}^{\prime}}$ se usa un puente diseñado adecuadamente, cuya señal de error, proporcional a dicha diferencia, se digitaliza para ser analizada posteriormente (10). Se usan pulsos de ondas a fin de evitar el calentamiento de la muestra (11).

El procedimiento de análisis de dichos datos se ha diseñado de forma que, en lo posible, no suponga la aceptación a priori de ninguna teoría. En la medida en que ello se consiga, la descripción que usemos será útil para abarcar un rango más amplio de materiales y situaciones (12).

Se asumen solamente tres ideas:

1) En general, las variables dependen del campo eléctrico instantáneo E

2) Dependen también de la amplitud del campo aplicado $\mathrm{E}_{0}$ de forma explícita $(13,14)$

3) Las distintas contribuciones al vector D debidas a distintos mecanismos son independientes

En función de ello el tratamiento de datos se efectúa de la forma siguiente:

El vector desplazamiento $\mathrm{D}(\mathrm{t})$, se considera igual a la suma de un término lineal y un término no lineal:

$$
D(t)=D_{L}(t)+D_{N L}(t)
$$

La parte no lineal $D_{\mathrm{NL}}$ se obtiene sin ambigüedad a partir de la integración de la señal de error del puente, y se desdobla en dos términos: uno es función única de $\mathrm{E}$, mientras que el otro representa las pérdidas.

$$
D_{N L}(t)=\widetilde{D}(E(t))+\Delta D(t)
$$

La derivada del primero respecto al campo nos da el incremento de la constante dieléctrica instantánea :

$$
\Delta \varepsilon\left(E, E_{0}\right)=\frac{1}{\varepsilon_{0}} \frac{d \tilde{D}\left(E, E_{0}\right)}{d E}=\Delta \varepsilon_{S}\left(E, E_{0}\right)+\Delta \varepsilon_{\mathrm{A}}\left(E, E_{0}\right)
$$

Éste se ha desdoblado en distintos términos; la parte antisimétrica y la simétrica, que a su vez se subdivide en $\boldsymbol{\varepsilon}_{\alpha}$, que es el valor de $\Delta \varepsilon$ para $\mathrm{E}=0$, y el término $\varepsilon_{\beta}$ que depende de $\mathrm{E}$.

$$
\Delta \varepsilon_{S}\left(E, E_{0}\right)=\varepsilon_{\alpha}\left(E_{0}\right)+\varepsilon_{\beta}\left(E, E_{0}\right)
$$

Si bien se puede analizar el comportamiento de estas contribuciones como funciones de E, en este trabajo nos centraremos fundamentalmente en el estudio de la parte $\varepsilon_{\alpha}$ y de los valores medios de $\varepsilon_{\beta}$ y de las pérdidas $\varepsilon^{\prime \prime}$.

\section{RESULTADOS Y DISCUSIÓN}

Este método de análisis ha sido aplicado a diversas muestras comerciales de Titanato Zirconato de Plomo del tipo duro (Vernitron PZT4) y blando (Ferroperm PZ27). Aunque la mayor parte de medidas se han realizado sobre muestras bien polarizadas y envejecidas, se han realizado también pruebas de envejecimiento y de despolarización y repolarización para analizar el efecto de dichos procesos y establecer las condiciones idóneas de medida. Se han aplicado campos cuya amplitud máxima es de 0,60 MV/m. La frecuencia ha sido fijada en $1000 \mathrm{~Hz}$.

\subsection{Dependencia explícita de $\mathrm{E}_{0}$}

Tal como se observa en las figuras 1 y 2, correspondientes a cerámicas blandas y duras, para cada experiencia la constante dieléctrica simétrica depende del campo instantáneo E, mientras que las curvas correspondientes a campos de distinta amplitud no coinciden, aumentando de manera progresiva a medida que aumenta la amplitud $\mathrm{E}_{0}$. Ello no ocurre con la parte antisimétrica, en la que se observa la superposición de las curvas (no dependen de la amplitud).

Se observa como las curvas están regularmente espaciadas en el material blando y con espaciamiento creciente en el duro, revelando una dependencia aproximadamente lineal o cuadrática, respectivamente.

\subsection{Dependencia de la fracción $\varepsilon_{\alpha}\left(\mathrm{E}_{0}\right)$}

\subsubsection{CON EL TIPO DE MATERIAL}

Para observar la dependencia y la importancia relativa de $\varepsilon_{\alpha}$ se ha representado simultáneamente con el incremento total de $\varepsilon^{\prime}$ (Figuras 3 y 4). Mientras que en los materiales blandos la fracción representa la casi totalidad de $\varepsilon^{\prime}$, en los duros pasa a ser la fracción $\varepsilon^{\prime}$ de menor peso. En el peor de los casos, el incremento de $\varepsilon_{\alpha}$ en el material duro ha llegado a ser como mucho 40 veces inferior de lo que se observa en los blandos. En esas curvas se manifiesta mucho mejor la dependencia lineal o cuadrática de los materiales blandos y duros respectivamente.

\subsubsection{CON EL ENVEJECIMIENTO}

La representación de $\varepsilon_{\alpha}$ y de $\varepsilon^{\prime}$ en función del logaritmo del tiempo transcurrido desde la alteración de la polarización en un material duro nos muestra que a pesar de su innegable evolución en el tiempo, su diferencia $\varepsilon_{\beta}$ apenas se ve alterada (Figura 5). Se puede observar como las pérdidas se van reduciendo a la par que el valor de $\varepsilon_{\alpha}$. Para muestras poco envejecidas, el término $\varepsilon_{\alpha}$ puede ser el más importante.

\subsubsection{CON LA POLARIZACIÓN}

Se ha tomado una muestra envejecida (A), se ha polarizado al revés (B), se la vuelve a polarizar en el sentido original apli- 


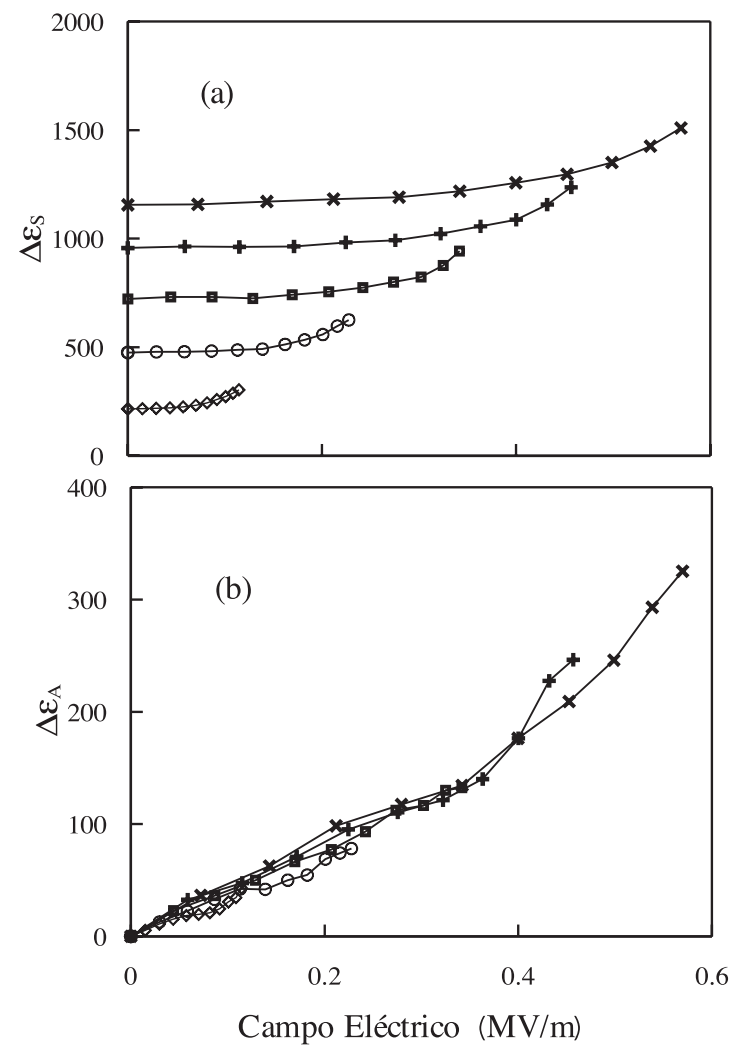

Figura 1. Parte simétrica (a) y antisimétrica (b) del incremento de la constante dieléctrica para distintas amplitudes $\mathrm{E}_{0}(0.11,0.23,0.35$, 0.46 , y $0.57 \mathrm{MV} / \mathrm{m}$ respectivamente) en un material blando (PZ27).
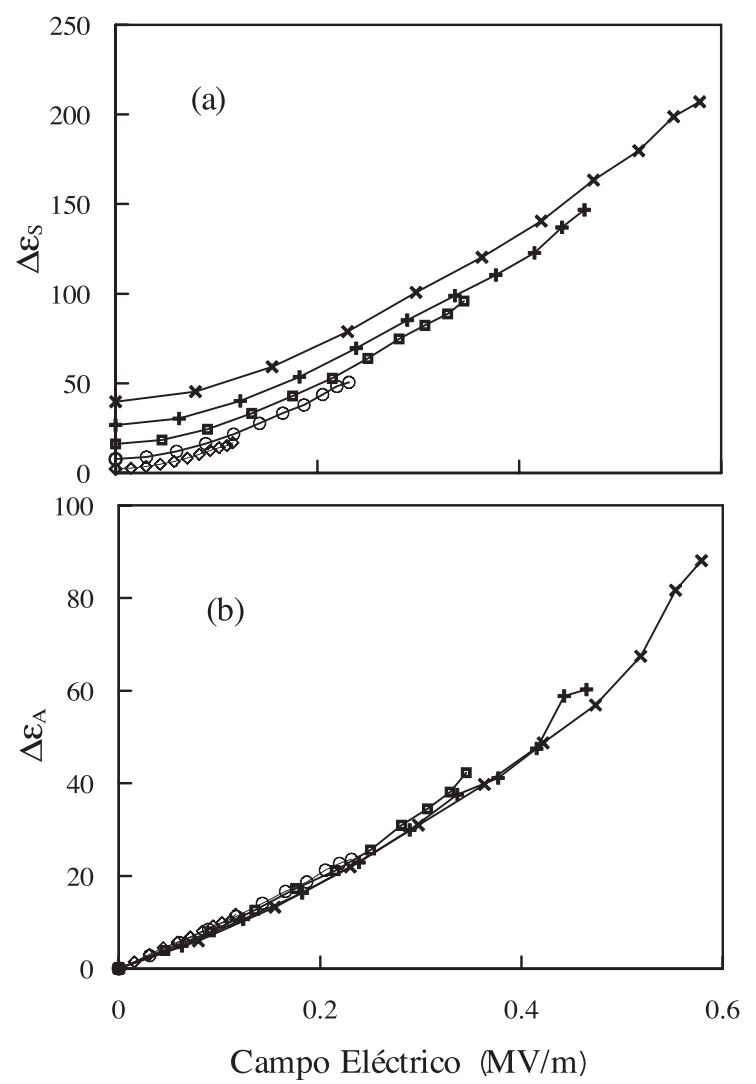

Figura 2.Parte simétrica (a) y antisimétrica (b) del incremento de la constante dieléctrica para distintas amplitudes $\mathrm{E}_{0}(0.11,0.23,0.35$, 0.46 , y $0.57 \mathrm{MV} / \mathrm{m}$ respectivamente) en un material duro (PZT4)

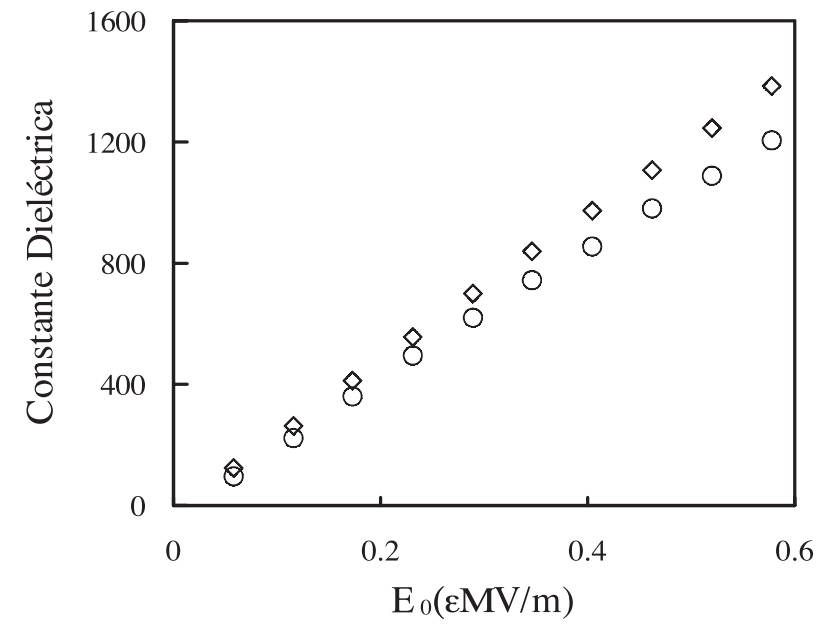

Figura 3. Incremento de la constante dieléctrica $\varepsilon^{\prime}(\diamond)$ y fracción $\varepsilon_{\alpha}$ (O) en función de la amplitud del campo aplicado en un material blando (PZ27).

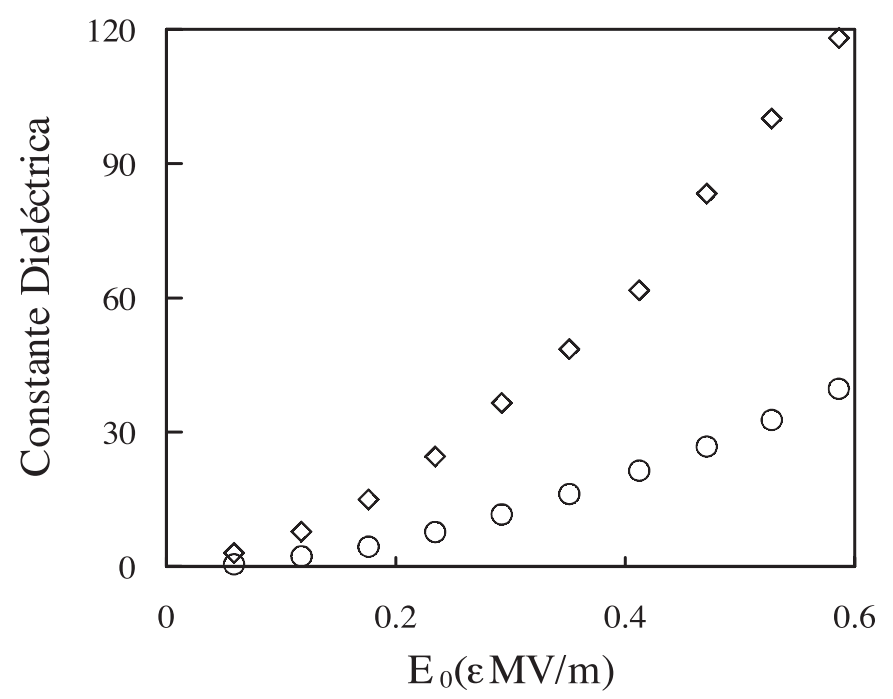

Figura 4. Incremento de la constante dieléctrica $\varepsilon^{\prime}(\diamond)$ y fracción $\varepsilon_{\alpha}$ (O) en función de la amplitud del campo aplicado en un material duro (PZT4).

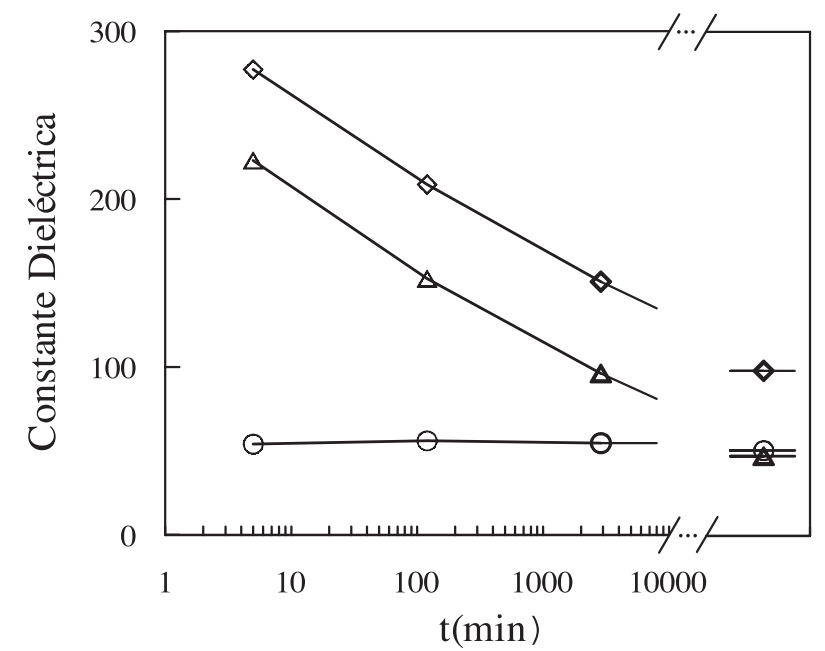

Figura 5. Evolución espontánea de $\varepsilon^{\prime}(\diamond), \varepsilon_{\alpha}(\Delta)$ y $\varepsilon_{\beta}=\varepsilon^{\prime}-\varepsilon_{\alpha}(O)$, medidas a $\mathrm{E}_{0}=0,6 \mathrm{MV} / \mathrm{m}$, después de un cambio en la polarización en un material duro. Se indica también su valor después de un tiempo muy largo (indeterminado). 


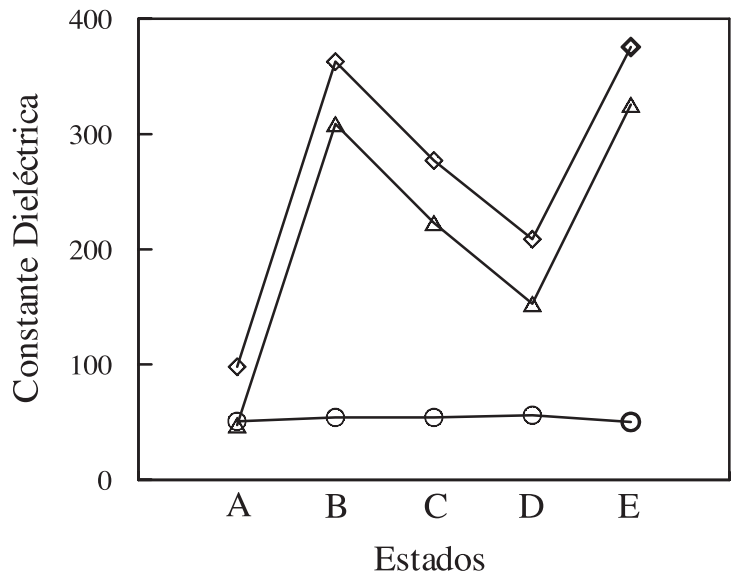

Figura 6. Evolución de $\varepsilon^{\prime}(\diamond), \varepsilon_{\alpha}(\Delta)$ y $\varepsilon_{\beta}=\varepsilon^{\prime}-\varepsilon_{\alpha}(O)$, para distintos estados de polarización en un material duro. $\mathrm{E}_{0}=0,6 \mathrm{MV} / \mathrm{m}$.

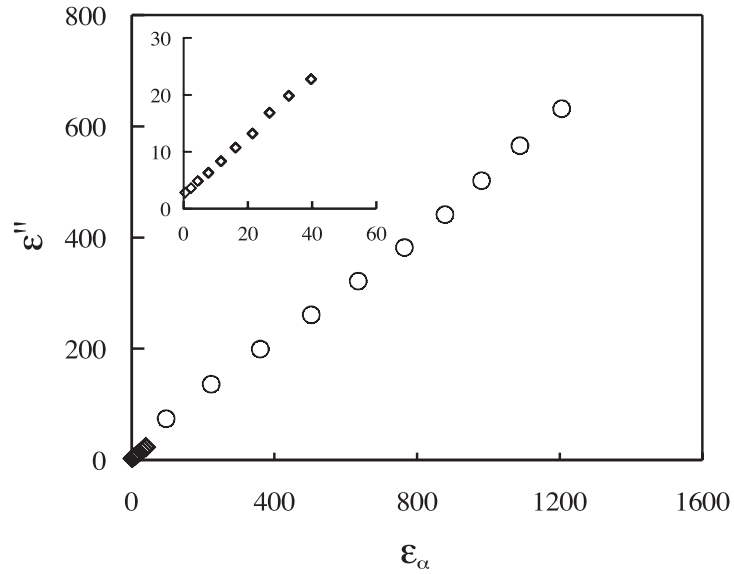

Figura 8. Dependencia de las pérdidas $\varepsilon^{\prime \prime}$ en función de $\varepsilon_{\alpha}$ para PZ27 (O) y PZT4 ( $\diamond)$

cando el campo durante un tiempo corto (C), se insiste en el mismo sentido, pero prolongando el tiempo (D) , y finalmente se le aplica un campo , controlando la carga, a fin de despolarizarla (E), comprobando mediante medidas de la impedancia que el acoplo piezoeléctrico es prácticamente nulo. En todas las etapas se deja reposar la muestra, cortocircuitándola, y se mide $\varepsilon_{\alpha}$ y $\varepsilon^{\prime}$. Se observa como, a pesar de las grandes variaciones de ambas magnitudes, la diferencia permanece inalterada (Figura 6). Es de suponer que en este proceso ha habido grandes cambios en la polarización de gran parte de los granos, lo que se refleja en la alteración de $\varepsilon_{\alpha}$. Sin embargo, no parece que $\varepsilon_{\beta}$ tenga nada que ver con el estado de polarización.

\subsection{Relación de las pérdidas con $\varepsilon_{\alpha}$}

$\mathrm{Al}$ representar las pérdidas $\varepsilon^{\prime \prime}$ en relación con $\varepsilon^{\prime}$ se obtiene una recta para cualquiera de los materiales, tal como muestra la figura 7. Ello nos indica que, en cualquier caso, la dependencia de las funciones $\varepsilon^{\prime}$ y $\varepsilon^{\prime \prime}$ con respecto a $\mathrm{E}_{0}$ sigue la misma ley. Sin embargo, la pendiente en el material blando es cercano a $1 / 2$, tal como se espera del modelo de Rayleigh, mientras que en el material duro es del orden de 1/6, o sea tres veces inferior. Si se representa $\varepsilon^{\prime \prime}$ en relación con $\varepsilon_{\alpha}$ (figura 8) se descubre que, con buena aproximación, las relaciones siguen siendo lineales, pero con pendientes muy semejantes. Se sospecha por tanto de que $\varepsilon_{\alpha}$ y $\varepsilon_{\beta}$ están asociados a mecanismos distintos, y de que la mayor parte de las pérdidas (si

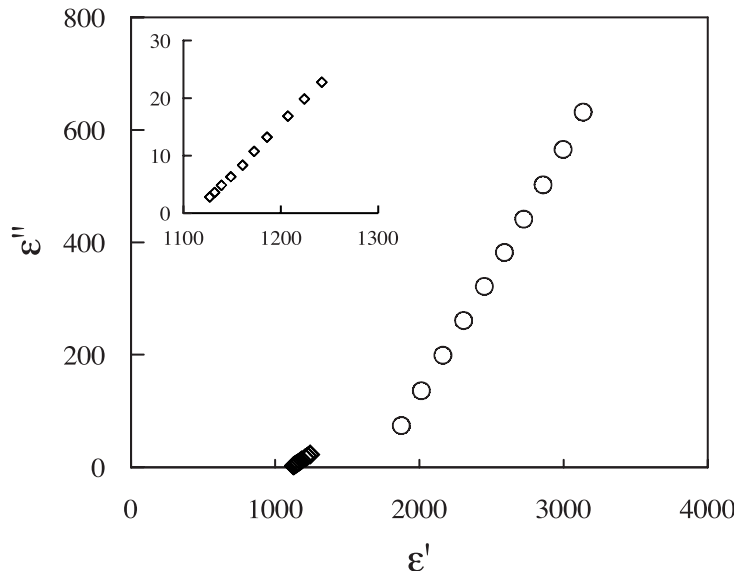

Figura 7. Dependencia de las pérdidas $\varepsilon^{\prime \prime}$ en función de $\varepsilon^{\prime}$ para PZ27 (O) y PZT4 ( $\diamond$ ). Detalle del comportamiento de PZT4.

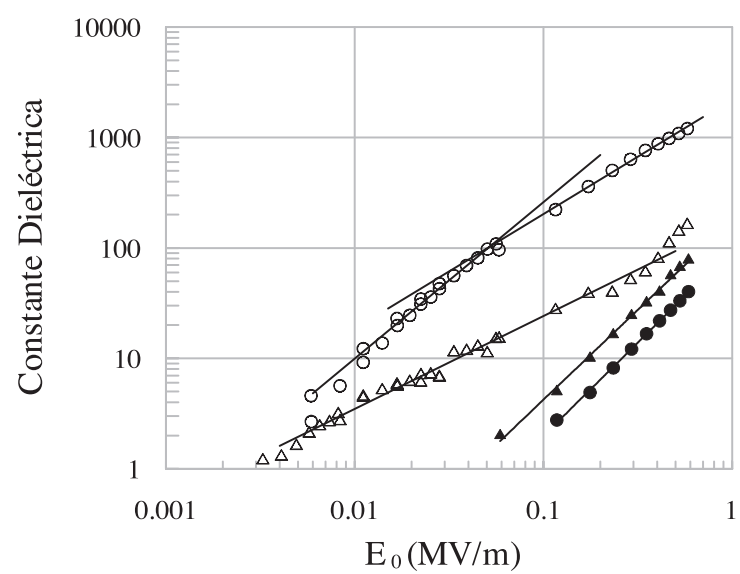

Figura 9. Representación en escala logarístimica de las componentes $\varepsilon_{\alpha}$ (círculos) y $\varepsilon_{\beta}$ (triángulos) en función de la amplitud de campo PZ27 $(\Delta \mathrm{O})$ y PZT4 $(\Delta$

no todas) corresponden al primer mecanismo, siendo este hecho coherente con el modelo de Rayleigh, a pesar de que la dependencia con el campo no siempre lo es.

\subsection{Dependencia potencial de $\varepsilon_{\alpha}$ con la amplitud del campo}

Para observar mejor la dependencia de $\varepsilon_{\alpha}$ y $\varepsilon_{\beta}$ con la amplitud del campo, se han representado en una escala logarítmica para ambos tipos de materiales (figura 9). Esto nos ha permitido abarcar en la misma gráfica un amplio rango de amplitudes.

Se observa en primer lugar la gran diferencia existente entre la no-linealidad de los materiales blandos y duros. En segundo lugar, mientras que la pendiente de ambas funciones es algo inferior a 2 para el material duro, la del material blando tiene una pendiente cercana a 1 en un amplio rango, mientras que el valor de $\varepsilon_{\alpha}$ muestra un cambio de pendiente, siendo ésta cercana a 1 por encima del umbral, y tendiendo a 1,5 para valores inferiores a él. Esto refuerza la idea de que existen dos mecanismos y de que el umbral afecta solamente a uno de ellos. El hecho de que las gráficas sean rectas en su mayor parte nos indica que la dependencia es de tipo potencial, tal como se indicaba en un trabajo anterior (15). No se descarta la posibilidad de que el distinto comportamiento entre los dos materiales pueda interpretarse simplemente por el hecho de tener los umbrales en zonas distintas, lo que implicaría que el material duro tendría el umbral por encima del valor de los campos utilizados usualmente. 


\section{CONCLUSIONES}

Se ha comprobado que se debe considerar la dependencia explícita de $\varepsilon$ con $\mathrm{E}$ y con $\mathrm{E}_{0}$, tanto en los materiales duros como en los blandos. Para un mismo material, tanto $\varepsilon$ como las pérdidas tienen la misma dependencia con $\mathrm{E}_{0^{\prime}}$ pero ésta es distinta en unos y otros materiales. No se aprecia dependencia explícita con $\mathrm{E}_{0}$ de la parte antisimétrica de $\varepsilon$.

En el modelo de Preisach, el sistema está definido por una función de distribución de los posibles biestables independientes que lo componen. Esta función se podría obtener, en principio, a partir de la función experimental $\varepsilon\left(E, E_{0}\right)$ en cualquiera de los materiales. A pesar de que la idea es muy atractiva, las funciones $\varepsilon\left(\mathrm{E}_{1} \mathrm{E}_{0}\right)$ de los materiales duros obtenidas no pueden derivarse, de forma inmediata, de ninguna distribución de este tipo, aun cuando puede interpretar razonablemente el comportamiento de las cerámicas blandas.(16)

La $\varepsilon_{\mathrm{NL}}$ puede desdoblarse en dos términos, de forma que uno de ellos, no dependa explícitamente del campo instantáneo. Se observa que la relación entre este término y las pérdidas casi no depende del tipo de material. Sin embargo, el segundo término no está asociado con pérdidas, ni depende de la polarización del material ni sufre los efectos del envejecimiento. Todo ello sugiere la coexistencia de dos mecanismos, claramente distintos, que contribuyen ambos a la nolinealidad de la cerámica.

La dependencia con la amplitud del campo de $\varepsilon_{\alpha}$ es en cualquier caso de tipo potencial. Sin embargo, el exponente depende del tipo de cerámica, siendo cercano a 1 en los blandos (dependencia casi lineal) y entre 1,6 y 2 en los duros ( casi cuadrática)

Se plantea la necesidad de analizar ambos términos de manera exhaustiva a fin de comprobar la generalidad de estas propuestas y de realizar experiencias específicas a fin correlacionar dichos términos con las características de las cerámicas ( composición, dopantes, tamaño de grano, etc. ) o con acciones aplicadas con posterioridad. Probablemente, ello nos daría información suficiente para determinar la naturaleza de ambos mecanismos, que es el objetivo último de este estudio.

\section{AGRADECIMIENTOS}

Este trabajo se inscribe dentro del proyecto TAP98-0911C03-02 CICYT.

\section{BIBLIOGRAFIA}

1. D. Damjanovic, M. Demartin, H.S. Shulman, M.Terstof, and N.Setter. "Instabilities in the piezoelectric properties of ferroelectric ceramics". Sensors Actuators A 53 , 353-360 (1996).

2. K. Uchino. "Recent trend of piezoelectric actuator developments". Proc ACTUATOR, P34 (2000).

3. S. Takahashi , Y. Sasaki, M. Umeda, K. Nakamura,and S. Uhea. "Nonlinear behavior in piezoelectric ceramic transducers". Proc. of the $12^{\text {th }}$ IEEE Int. Sym. on Appl. of Ferroelectrics, 11-16 (2000)

4. V. Mueller and H.Beige. "Nonlinearity of soft PZT piezoceramic for shear and torsional actuator applications". Proc. of the $11^{\text {th }}$ IEEE Int. Sym. on Appl. of Ferroelectrics, 459-462 (1998).

5. U.Robels, J.H. Calderwood,and G.Arlt. "Shift and Deformation of the histeresis curve of ferroelectrics by defects: An electrostatic model". J. Appl. Phys. 77, 4002-4008 (1995).

6. Q.M. Zhang, H. Wang, N. Kim,and L.E. Cross. “Direct evaluation of domainwall and intrinsic contributions to the dielectric and piezoelectric response and their temperature dependence on lead zirconate-titanate ceramics". J. Appl. Phys. 75, 454-459 (1994).

7. S. Li, W. Cao, and L.E.Cross. "The Extrinsic nature of Nonlinear behavior observed in lead zirconate titanate ferroelectric ceramic". J. Appl. Phys 69, 7219-7224 (1991).

8. D.Damjanovic. "Stress and frequency dependence of the direct piezoelectric effect in ferroelectric ceramics". J. Appl. Phys 82, 1788-1797 (1997).

9. V. Mueller, E. Fuchs, and H. Beige. "Characterization of nonlinear properties of soft PZT- Piezoceramics". Ferroelectrics 240, 67-74 (2000)

10. R. Pérez, E. Minguella y J.E.García. "Puente parta el análisis dieléctrico no lineal de cerámicas piezoelectricas". Proc SAAEI'00 Terrassa, 237-240 (2000).

11. D.A.Hall. "Rayleigh behaviour and the threshold field in ferroelectric ceramics". Ferroelectrics 223, 319-328 (1999).

12. J.E. García, R. Pérez, and A. Albareda. "High electric field measurement of dielectric constant and losses of ferroelectric ceramics". J. Phys. D: Appl. Phys. 34, 3279-3284 (2001).

13. P.J. Stevenson and D.A.Hall . "The effect of grain size on the high field dielectric properties of hard PZT ceramics". Ferroelectrics 223, 309-318 (1999).

14. P. Gonnard, V. Perrin, M.Trocaz. "Non-linearites du coefficient piezoelectrique inverse sous champ electrique eleve". $3^{\text {emes }}$ Journées d'etudes. Materiaux et Composantes Piezo-piro-ferroelectriques, P2 (1999).

15. R. Perez, J.E. Garcia, and A.Albareda. "Nonlinear dielectric behavior of piezoelectric ceramics". Proc. of the $12^{\text {th }}$ IEEE Int. Sym. on Appl. of Ferroelectrics, 443-446 (2000) .

16. D. Damjanovic, G. Robert, J. Muller, M. Demartin, D. V. Taylor, and N. Setter. "From ferroelectric ceramics and single crystals to thick and thin films: how domain-wall processes control the piezoelectric and dielectric properties". Proc. of the $12^{\text {th }}$ IEEE Int. Sym. on Appl. of Ferroelectrics, 305310 (2000).

Recibido: 31.05 .01

Aceptado: 10.12 .01 